Mens

revue d'histoire intellectuelle de l'Amérique française

Véronique Auzépy-Chavagnac. Jean de Fabrègues et la Jeune Droite Catholique. Aux sources de la Révolution nationale. Préface de René Rémond, Villeneuve d'Ascq (Nord), Presses universitaires du Septentrion, 2002.464 p.

\title{
Christian Roy
}

Volume 5, numéro 1, automne 2004

URI : https://id.erudit.org/iderudit/1024398ar

DOI : https://doi.org/10.7202/1024398ar

Aller au sommaire du numéro

Éditeur(s)

Centre de recherche en civilisation canadienne-française

ISSN

1492-8647 (imprimé)

1927-9299 (numérique)

Découvrir la revue

Citer ce compte rendu

Roy, C. (2004). Compte rendu de [Véronique Auzépy-Chavagnac. Jean de Fabrègues et la Jeune Droite Catholique. Aux sources de la Révolution nationale. Préface de René Rémond, Villeneuve d'Ascq (Nord), Presses universitaires du Septentrion, 2002. 464 p.] Mens, 5(1), 195-204. https://doi.org/10.7202/1024398ar d'utilisation que vous pouvez consulter en ligne. 
Véronique Auzépy-Chavagnac. Jean de Fabrègues et la Jeune Droite Catholique. Aux sources de la Révolution nationale. Préface de René Rémond, Villeneuve d'Ascq (Nord), Presses universitaires du Septentrion, 2002. 464 p.

Dans un article publié en 1995 ("La Jeune Droite Catholique (années 1930 et 1940) : histoire d'une différence ", Mil neuf cent. Revue d'bistoire intellectuelle. Numéro spécial sur le thème "Les intellectuels catholiques. Histoire et débats »), Véronique Auzépy-Chavagnac (qui enseigne aujourd'hui à l'Institut Catholique de Paris) avait admirablement démêlé les parcours politiques et éditoriaux des intellectuels "nonconformistes » de droite dans le contexte des mutations du catholicisme français. Il s'agissait en fait du résumé d'une thèse pour l'Institut d'études politiques, dirigée par René Rémond et centrée sur Jean de Fabrègues, en tant que digne représentant d'une tradition intellectuelle catholique de droite dont celui-ci n'aura rien ignoré des heurs et malheurs — de sa position dominante dans l'Église française du premier aprèsguerre à sa marginalisation post-conciliaire. La période couverte en profondeur dans la thèse s'arrêtait à l'été 1941, mais dans cette version publiée, le tableau est complété par un nouveau chapitre sur la période de l'Occupation et un épilogue survolant les activités subséquentes de Fabrègues, qui dirigea La France catbolique de 1945 à 1970 - y tenant tête au progressisme de la revue rivale Témoignage chrétien - et publia une quinzaine d'essais et de biographies. Certains de ces ouvrages étaient consacrés à ses propres inspirateurs. Au premier rang de ceux-ci se trouvent ainsi Marc Sangnier et Charles Maurras (dont il fut même le secrétaire juste avant une douloureuse rupture en 1930), puisqu'il avait toujours cherché à " réconcilier l'esprit du Sillon et celui de l'Action Française " en mariant la charité apostolique du catholicisme social au 
réalisme politique de la tradition monarchique (p. 85). Il faut encore citer les romanciers Georges Bernanos et François Mauriac, figures tutélaires respectives de Réaction (1930-32) et de La Revue du Siècle (1933-34), de même que les philosophes Gabriel Marcel et Jean Guitton, qui joueraient ce rôle pour Civilisation (1938-39) puis Demain (1942-44) respectivement, parmi les maintes revues qu'anima Jean de Fabrègues. Par sa "synthèse baroque d'un catholicisme intransigeant et d'une large ouverture intellectuelle » (p. 406), celui-ci sut toujours rassembler un vaste éventail de collaborations de tous horizons pour discuter des grands enjeux de civilisation dans leur portée spirituelle et politique.

Ne fût-ce qu'à ce titre, Jean de Fabrègues joua un rôle de cheville ouvrière dans les recompositions successives de la nébuleuse des «non-conformistes des années 30 » d'abord recensée par Jean-Louis Loubet del Bayle en 1969 dans son ouvrage classique de ce titre. Si ces jeunes intellectuels y étaient répartis entre les trois courants principaux d'Esprit, de l'Ordre Nouveau et de la Jeune Droite, l'originalité de Chavagnac est de prétendre distinguer du sein de cette dernière une Jeune Droite Catholique, pour l'opposer à la Jeune Droite tout court, dite parfois non-conformiste, à propos de laquelle elle renvoie en passant à Nicolas Kessler pour son Histoire politique de la Jeune Droite (1929/1942): Une révolution conservatrice à la française, ouvrage paru chez L'Harmattan en 2001, dont elle ne s'est donc pas servi dans sa thèse. Cette innovation de sa part ne convainc qu'à demi, puisqu'elle ne concerne guère que la séquence compliquée des dissidences plus ou moins marquées et nuancées envers la hiérarchie catholique et / ou l'Action Française au lendemain de la condamnation de cette dernière par le Saint-Siège en 1926. Cette décision forçait les Catholiques de droite à choisir entre Maurras et saint Thomas d'Aquin, alors qu'ils avaient pris l'habitude de souder au nom de la droite raison leurs systèmes res- 
pectifs en une même machine de guerre : celle de l'« ordre social chrétien » à reconquérir sur la République laïciste. Plus généralement, elle était dirigée contre le " désordre démocratique » et libéral exacerbé dans l'américanisme et le communisme d'abord, dans le fascisme et l'hitlérisme ensuite. Or, à mesure que l'on avance dans le récit détaillé des constants chassés-croisés politiques et éditoriaux entre intellectuels nationalistes - croyants ou non - issus des mouvances initialement distinctes de Jean Maxence et de Jean de Fabrègues, on tend à perdre le fil de ce qui ferait la cohésion de sa Jeune Droite Catholique face à une "autre " Jeune Droite, moins soucieuse de politique chrétienne que de politique tout court et volontiers révolutionnaire - alors que Fabrègues prétend réagir à l'oubli de la métaphysique en modernité en se tournant vers le passé. D'où le titre de sa revue Réaction pour l'Ordre lancée en 1930 comme la première des revues non-conformistes; avec certains des autres titres d'abord envisagés comme L'Esprit réactionnaire et L'Ordre, celui-ci permet déjà de discerner tant les préoccupations rapprochant cette Jeune Droite de ces principaux autres mouvement non-conformistes dont les organes s'appelleront bientôt Esprit et L'Ordre Nonvean que les nostalgies qui la distingue de ces derniers.

Ce sont peut-être les comparaisons fréquentes entre non-conformismes de gauche et de droite qui sont ce qu'il y a de plus stimulant ici - surtout dans le cadre du catholicisme. Toutefois, Chavagnac semble tout ignorer de la grande diversité des sources du personnalisme français, et s'en tient au récit canonique des milieux catholiques (de plus en plus battu en brèche par la recherche récente), qui n'y voit qu'une transmission directe et tardive de Maritain à Mounier. Elle n'en met pas moins en évidence une filiation qui en surprendra plus d'un, dans la transmission précoce d'un incontestable discours personnaliste de droite du Maritain maurrassien au jeune Fabrègues dès 1927-28. Celui-ci invoque alors saint 
Thomas pour opposer à l'État tout-puissant de l'Antiquité et de la modernité « la dignité essentielle de la personne humaine commune à tous » reconnue par le XIII ${ }^{\mathrm{e}}$ siècle chrétien, que bafouent l'esclavage et la condition prolétarienne - même glorifiée, puisque «là encore la logique marxiste pousse à ses dernières conséquences la grande tradition individualiste » (cité pp. 123, 125) en faisant du genre humain un Dieu immanent. Défendant La Primauté du Bien commun contre les personnalistes comme Maritain et Mounier au moment où leurs idées avancées commencent à faire leur chemin dans le monde catholique durant la guerre, Charles De Koninck, professeur de philosophie à l'Université Laval, les accusera justement de faire le jeu de cet «ordre nouveau » marxiste que préparerait l'humanisme libéral. Il refuse implicitement leur distinction (spécieuse aux yeux de bien d'autres critiques thomistes) de l'individu et de la personne, d'abord introduite en 1925 dans Trois réformateurs par Jacques Maritain, pour qui «"la cité chrétienne est aussi foncièrement anti-individualiste que foncièrement personnaliste." La Jeune Droite Catholique s'accorde à ce point de vue. "L'individu n'est rien, la personne est tout", proclame Jean de Fabrègues. » Dans une lettre à Charles Maurras du 10 mars 1932, Fabrègues renchérit en invoquant la distinction parallèle (sur laquelle Charles De Koninck insistera "contre les personnalistes" en les accusant d'un individualisme qu'ils récusaient pourtant tous !) «entre les biens spirituels, qui s'accroissent en partage, et ceux qui, matériels, ne sauraient être partagés sans diminution. Hier encore, votre distinction de l'individu et de la personne [...] À ce propos, il me semble que le point de vue de la personne une fois substitué à celui de l'individu, l'opposition disparaît entre le point de vue du sujet et celui du gouvernement-objet. L'intérêt général ou bien commun, en effet, n'est autre que l'intérêt, non de l'individu mais de la personne, c'est à dire de ce qu'il y a de plus constant, élevé, libérant, dans la matière en nous. » (p. 
185) Emmanuel Mounier ne dira pas autre chose quand viendra le moment d'expliquer en quoi «l'individu n'est pas la personne» - à partir de 1934.

Sauf que "pour Fabrègues, la nation est le prolongement de la personne, son aire naturel [sic] d'expression, » (p. 186) et «seul un Roi peut unir le ciel et la terre, constituer le lien tant souhaité du spirituel et du temporel», selon "l'idéal contre-révolutionnaire et maurrassien qui est le sien : n'incarne-t-il pas la personne opposée à l'individu, par cette fonction royale qui dépasse l'homme et n'est-il pas le seul à pouvoir ainsi symboliser la continuité de la nation? » (p. 191) Or "l'autorité royale ne saurait assurer le bien commun sans le relais des corporations", "cadres sociaux naturels (de) la personne humaine libre » d'après le manifeste du groupe de Réaction, convaincu que «l'organisation corporative de l'économie et de la société permettrait de décentraliser le pouvoir et de limiter l'intervention de l'État. » (p. 192) De plus, «selon Fabrègues, l'ordre politique ne saurait être respectueux de la personne sans la reconnaissance officielle de la vérité catholique » par un "État théologico-politique » « appuyant sa légitimité et son rôle sur les valeurs du christianisme reconnues pour telles. Pour Mounier en revanche, l'ordre humain est naturellement ordonné à la vie chrétienne. Il n'a besoin pour devenir ce qu'il est que du témoignage personnel de chacun" (p. 213) dans le cadre d'un nouvel humanisme «à géométrie variable ", ouvert au spirituel, mais ne cherchant pas à en imposer une définition canonique par le biais d'un pouvoir politique que celle-ci sanctionnerait.

Malgré ce qu'en disent des travaux comme ceux de J.M. Mayeur et d'E. Poulat, qui ont mis en relief l'héritage intransigeant d'antimodernisme au sein même du catholicisme social (fût-ce aux dépens de dimensions libérales qui ne relèvent pas forcément de la seule tactique politique), Chavagnac 
estime « qu'à cette conception ouverte et conciliante du christianisme s'oppose la vision catholique intransigeante de Fabrègues » (p. 214).

Tandis que Mounier fait confiance à l'homme, Fabrègues se défie de son péché. [...] Cette différence d'appréciation de la nature humaine se traduit dans l'ordre politique. Puisque l'homme est pécheur pense Fabrègues, sa liberté ne peut se construire sans l'aide d'institutions parées d'une certaine autorité. Puisque l'homme est bon, parie Mounier, un ordre chrétien naîtra nécessairement du respect de sa liberté. (p. 227)

On sait aujourd'hui grâce aux travaux d'E.-Martin Meunier et de Jean-Philippe Warren que tel fut bien le pari des militants chrétiens progressistes québécois qui ont fourni «l'horizon personnaliste de la Révolution tranquille » — avec des résultats ambigus qui ont récemment amené bien des observateurs à déplorer la perte dans son sillage de toutes balises, de tous repères et du sens même de l'institution chez l'individu postmoderne ne voyant en celle-ci (comme y tendaient parfois certains personnalistes tels Nicolas Berdiaev) qu'hétéronomie objectivante entravant le libre épanouissement de son autonomie créatrice.

Force est d'admettre que de tels développements ont de quoi conférer une résonance étrangement prophétique aux précoces avertissements d'un personnaliste chrétien de droite dont Chavagnac démontre que pour lui

la protection de la véritable liberté humaine nécessite : "des remparts [...], les corps sociaux, les institutions, l'autorité [...]. Où sont les vôtres? Toutes vos sympathies affichées nous laissent à penser qu'elles ne sont pas sorties de ce plan de confiance dans la liberté humaine qui a conduit l'homme où il est ", s'exclame Fabrègues à l'adresse de Mounier. (p. 235) 
Au même moment, face aux émeutes antiparlementaires de février 1934 et à la polarisation politique qui en résulte, «ces frères ennemis dans le catholicisme [...] voient se préciser leurs oppositions » (p. 247), selon l'une de ces éclairantes mises en parallèle qui sont un leitmotiv du livre de Chavagnac, et qui amènent la question - formulée en conclusion - de savoir si « on peut ou non parvenir à réconcilier les expressions de la gauche et de la droite de catholicisme français » (p. 407) Ainsi que l'expose Chavagnac:

Il s'avère que leur vision du monde respective repose [sic] sur une conception différente de la nature humaine : double pour Fabrègues qui souligne la tentation permanente du corps pour l'esprit; une aux yeux de Mounier pour qui l'homme, dans une perspective d'amélioration, est toute [sic] aspiration vers le spirituel.

De cette opposition d'un pessimisme et d'un optimisme chrétiens surgit une plus forte demande institutionnelle de la part de Fabrègues que de celle de Mounier. De là le reproche d'« angélisme » du premier au second et le retrait d'Esprit devant le réalisme politique de la Jeune Droite Catholique. Leur rejet jusque-là commun de la démocratie parlementaire, qui s'exprimait dans le slogan ni droite, ni gauche, s'estompe dès lors au profit d'une sympathie inverse pour l'un des deux camps. À droite, Fabrègues apprécie les valeurs d'autorité et de hiérarchie sans lesquelles n'existe pas, à ses yeux, de véritable liberté. Le programme politique de la Revue $d u X X^{e}$ siècle suggère la naissance d'une monarchie personnaliste. À gauche, Mounier admire la confiance dans le libre-arbitre et la recherche d'égalité. Son évolution annonce la proposition de démocratie personnaliste qui sera celle d'Esprit à la veille de la guerre (p. 247).

Ce « débat autour du sens à donner à la notion de personnalisme » (p. 322) continue alors de diviser ces deux mou- 
vances d'intellectuels catholiques à un moment où elles n'ont jamais été si proches. En effet, puisque la III ${ }^{\mathrm{e}}$ République se montre aussi incapable de faire face au péril imminent qu'indigne de lui survivre, elles réclament toutes deux une Révolution nationale pour changer le régime avant qu'il ne soit trop tard, donnant ainsi à la France une chance de tenir tête à l'Allemagne et de préserver la civilisation occidentale de la barbarie. Conçue avant la guerre pour lui faire halte par une victoire militaire et morale, la Révolution nationale naîtra plutôt dans le contexte de la défaite et deviendra peu à peu le vecteur des pires démissions de l'esprit français, au grand dam de maints de ses acteurs. Si Mounier et ses amis font un moment de l'entrisme dans les nouvelles institutions culturelles telles que Jeune France (où lui-même lance les Maisons de la Culture), c'est non sans chercher à donner l'air de rien une transcription personnaliste des slogans d'un régime dont ils craignent certaines tendances dictatoriales, et à fortifier sous son nez des espaces de pluralisme et de réflexion doctrinale. Fabrègues quant à lui s'oppose à leur influence en s'appuyant sur « une droite dure qu'il sait avant tout patriote» (p. 347). Dans sa diatribe contre Mounier, Fabrègues estime alors que

parler de la liberté, même s'il s'agit de celle de la personne humaine, n'est envisageable que dans le cadre d'une nation unie et organisée. Dans les circonstances où se trouve le pays, la communauté personnaliste de Mounier s'oppose à la communauté nationale que veut construire Fabrègues : disciplinée, soumise à une autorité soucieuse du Bien commun. (p. 353)

Ceci «place les institutions au centre du débat politique » (comme au temps où les sillonistes prônaient l'évolution des institutions par une réforme morale et les maurrassiens la conversion morale par un changement d'institutions) entre " deux systèmes de pensée qui, pour s'appuyer sur la même foi chrétienne, s'affrontent autour d'une vision plus ou moins 
charnelle ou idéale de la communauté française. C'est en défendant la dignité de la personne que Mounier entend protéger la communauté nationale, en défendant la communauté nationale que Fabrègues entend protéger la dignité de la personne. » (p. 356) (Notons en passant que cette différence d'approches personnalistes trouvera une sorte de parallèle au Québec dans la tension entre les courants libéraux fédéralistes et néo-nationalistes parmi les artisans de la Révolution tranquille, qui en viendront de plus en plus à s'affronter sur le sens et les orientations à lui donner.) Jean de Fabrègues donne alors le primat aux considérations politiques pour incarner le spirituel par la présence indéfectible du témoignage chrétien du «bon » côté, comme le fera bientôt à son tour Mounier de l'autre bord, avec un aveuglement plus acharné encore quant aux réalités du communisme. Mounier tomba ainsi à la Libération dans un paradoxe semblable à celui que souligne J.-L. Loubet del Bayle à propos des "héritiers de la Jeune Droite" à Vichy dans la nouvelle conclusion mise à jour de ses Nonconformistes des années 30 (Seuil, 2001, p. 482) : "vouloir réaliser une révolution à finalité "communautaire" et non-étatiste par des moyens étatiques ».

C'est ce qu'a mis en lumière avec autant de pénétration que de sens des nuances Véronique Auzépy-Chavagnac, dans une synthèse qui débouche sur ce simple constat: «Mounier est séduit par le souci de justice sociale qu'il sent dans le communisme, Fabrègues par l'ordre communautaire qu'il attend de Vichy"; dans les deux cas, "les milieux de pouvoir dans lesquels ils s'introduisent pour faire progresser leurs idées les englobent dans leurs querelles » et "en déforment le véritable enjeu » chrétien en le faisant passer au filtre de la justification politique (p. 404). Ceci soulève la question de savoir s'il y avait moyen pour un chrétien de se faufiler entre les mailles de ce piège. En guise de réponse, rappelons pour finir 
qu'il reviendra à Bernanos de refuser la justification sous toutes ses formes en sacrifiant l'engagement militant à sa seule vocation de prophète (ainsi qu'il l'explique à Alexandre Marc quand celui-ci cherche à le gagner à sa troisième voie personnaliste fédéraliste), puisque cette figure estimée de Fabrègues comme de Mounier sut renvoyer dos à dos les complaisances qu'ils ont chacun favorisées envers ces idoles de l'Ordre social et de la Justice sociale auxquelles ont successivement sacrifié les intellectuels catholiques avant et après la guerre.

Christian Roy Facultés de théologie et de philosophie Université Laval 